\title{
Does the placebo effect modulate drug bioavailability? Randomized cross-over studies of three drugs
}

\author{
Muhammad M Hammami ${ }^{1,2^{*}}$ D, Ahmed Yusuf ${ }^{1}$, Faduma S. Shire ${ }^{1}$, Rajaa Hussein ${ }^{1}$ and Reem Al-Swayeh ${ }^{1}$
}

\begin{abstract}
Background: Medication effect is the sum of its drug, placebo, and drug*placebo interaction effects. It is conceivable that the interaction effect involves modulating drug bioavailability; it was previously observed that being aware of caffeine ingestion may prolong caffeine plasma half-life. This study was set to evaluate such concept using different drugs.
\end{abstract}

Methods: Balanced single-dose, two-period, two-group, cross-over design was used to compare the pharmacokinetics of oral cephalexin, ibuprofen, and paracetamol, each described by its name (overt) or as placebo (covert). Volunteers and study coordinators were deceived as to study aim. Drug concentrations were determined blindly by in-house, high performance liquid chromatography assays. Terminal-elimination half-life ( $\left.\mathrm{t}_{1 / 2}\right)$ (primary outcome), maximum concentration $\left(C_{\max }\right), C_{\max }$ first time $\left(T_{\max }\right)$, terminal-elimination-rate constant $(\lambda)$, area-underthe-concentration-time-curve, to last measured concentration $\left(A \cup C_{T}\right)$, extrapolated to infinity $\left(A \cup C_{1}\right)$, or to $T_{\max }$ of overt drug ( $A \cup C_{\text {Overttmax }}$ ), and $C_{\max } / A \cup C_{1}$ were calculated blindly using standard non-compartmental method. Covert-vs-overt effect on drug pharmacokinetics was evaluated by analysis-of-variance (ANOVA, primary analysis), $90 \%$ confidence interval (Cl) using the $80.00-125.00 \%$ bioequivalence range, and percentage of individual pharmacokinetic covert/overt ratios that are outside the $\pm 25 \%$ range.

Results: Fifty, 30, and 50 healthy volunteers (18\%, 10\%, and 6\% females, mean (SD) age 30.8 (6.2), 31.4 (6.6), and 31. 2 (5.4) years) participated in 3 studies on cephalexin, ibuprofen, and paracetamol, respectively. Withdrawal rate was $4 \%, 0 \%$, and $4 \%$, respectively. Eighteen blood samples were obtained over 6,10 , and $14 \mathrm{~h}$ in each study period of the three drugs, respectively. ANOVA showed no significant difference in any pharmacokinetic parameter for any of the drugs. The $90 \% \mathrm{Cls}$ for $A \cup C_{T}, A \cup C_{1}, C_{\max }, A \cup C_{\text {Overttmax, }}$ and $C_{\max } / A \cup C_{1}$ were within the bioequivalence range, except for ibuprofen $C_{\max }$ (76.66-98.99), ibuprofen $C_{\max } / A \cup C_{1}$ (77.19-98.39), and ibuprofen (45.32-91.62) and paracetamol (51.45-98.96) AUC Overttmax. Out of the 126 individual covert/overt ratios, 2.0-16.7\% were outside the $\pm 25 \%$ range for $A \cup C_{T}, 2.0-4.2 \%$ for $A \cup C_{1}, 25.0-44.9 \%$ for $C_{\max }, 67.3-76.7 \%$ for $A \cup C_{\text {Overttmax, }}$ and $45.8-71.4 \%$ for $T_{\max }$.

Conclusions: This study couldn't confirm that awareness of drug ingestion modulates its bioavailability. However, it demonstrates the trivial effect of blinding in bioequivalence studies and the extent of bio-variability that would be expected when comparing a drug product to itself.

Trial registration: ClinicalTrials.gov identifier: NCT01501747 (registered Dec 26, 2011).

Keywords: Placebo effect, Bioavailability, Plasma terminal half-life, Pharmacokinetic parameters, Cephalexin, ibuprofen, Paracetamol

\footnotetext{
* Correspondence: Muhammad@kfshrc.edu.sa

${ }^{1}$ Clinical Studies and Empirical Ethics Department, King Faisal Specialist

Hospital and Research Center, P O Box \# 3354 (MBC 03), Riyadh 11211, Saudi

Arabia

${ }^{2}$ Alfaisal University College of Medicine, Riyadh, Saudi Arabia
} 


\section{Background}

The placebo effect has been utilized in medical practice since antiquity and continues to be commonly used [1]. Changes over time in the placebo arm of a randomized, double-blind, placebo-controlled trials don't separate the placebo effect (meaning response) [2] from methodological factors such as regression to the mean, natural course, and the Hawthorne effect. [3] Although it was once argued that if a placebo effect exists it would be of negligible importance [4], under some circumstances, the placebo effect could be clinically important $[5,6]$ and comparable in size to the drug effect [7-11]. The placebo effect may explain why generic drug products that pass rigorous bioequivalence tests are perceived as less potent (not more potent) than their more expensive branded counterparts [12, 13].

Differences between drug and placebo arms in clinical trials may represent not only drug pharmacological effect but also a drug* placebo interaction effect and thus may underestimate [7, 14] or overestimate [8] pharmacological drug effect. The possibility of a negative interaction effect may explain the clinically trivial effect of antidepressants as deduced from clinical trials [15].

It was suggested since the 1950s that the effect of false belief may countermand the effects of active drugs [16]. A neuro-imaging study showed that alcohol intoxication and expectancy have opposite effects on neuronal activation [17]. The possibility that the drug* placebo interaction effect may involve modulation of drug bioavailability has not been well explored. Theoretically, it is possible that the interaction effect may involve altering gastric emptying, intestinal transit time, or drug elimination. Previously, we conducted a $14 \mathrm{~h}$ bioavailability study on 22 volunteers who received caffeine described as caffeine or as placebo in a balanced randomized cross-over design. Mean plasma caffeine levels were consistently lower in the terminal part of the concentration-time curve, caffeine area-under-thetime-concentration curve (AUC) was significantly lower, and plasma caffeine terminal half-life was significantly shorter after receiving caffeine described as placebo [7], suggesting the importance of blinding in bioequivalence studies which compare generic and brand drug products and in clinical trials even with objective endpoints.

The rate and extent of drug bioavailability are commonly evaluated by maximum concentration $\left(C_{\max }\right)$ and AUC to last measured concentration $\left(\mathrm{AUC}_{\mathrm{T}}\right)$ or extrapolated to infinity $\left(\mathrm{AUC}_{\mathrm{I}}\right)$, respectively, using the noncompartmental method. The rate of bioavailability can be also evaluated by first time of $C_{\max }\left(T_{\max }\right)$, the ratio $\mathrm{C}_{\text {max }} / \mathrm{AUC}_{\mathrm{I}}$, and $\mathrm{AUC}$ to $\mathrm{T}_{\max }$ of reference drug $\left(A U C_{\text {Reftmax }}\right)$. Average bioequivalence $(\mathrm{ABE})$ between a test and reference products of the same drug, the standard worldwide requirement to market generic drug products, is demonstrated if the $90 \%$ confidence interval
(CI) on their $\mathrm{C}_{\text {max }}, \mathrm{AUC}_{\mathrm{T}}$, and $\mathrm{AUC}_{\mathrm{I}}$ geometric mean ratio is in the range $80.00-125.00 \%[18,19]$.

Several concerns have been raised regarding the $\mathrm{ABE}$ standards, including, using relatively wide bioequivalence limits and being unable to ensure therapeutic equivalence in all subjects; several individual pharmacokinetic ratios can fall well outside the $\pm 20 \%$ range despite demonstrating ABE. [18-20] Intra-subject variability is commonly estimated by intra-subject coefficient of variation $(\mathrm{CV})$. Large intra-subject $\mathrm{CV}$ can be due to intra-drug variability (firstpass or metabolic variability, gastric emptying, etc.), intraproduct variability (tablet to tablet or batch to batch), inter-product variability (generic vs. reference product), or subject-by-product interaction (i.e., the difference between products is not the same across subjects). Large intrasubject variability is especially important for narrow therapeutic index (NTI) drugs, for which individual bioequivalence (IBE) model, 75/75 rule, and intra-subject variability comparisons have been advocated [21-23]. The 75/75 rule requires $\geq 75 \%$ of individual test-reference pharmacokinetic ratios to be within $\pm 25 \%$.

We hypothesized that the drug*placebo interaction effect may involve modulation of drug pharmacokinetics. We elected to study two over-the-counter medicines, ibuprofen and paracetamol, because of their expected familiarity to study volunteers (and hence potentially having a placebo effect) and cephalexin as a "negative control" because of its expected unfamiliarity. We were not able to confirm our previous observation in any of the three drugs. However, we used the data to explore the extent of bio-variability that can be observed when comparing a drug product to itself.

\section{Methods \\ Design}

Volunteers were consecutively assigned to one of three randomized, two-period, two-sequence, cross-over studies, using cephalexin, ibuprofen, or paracetamol. In each study, the volunteers received one of the three drugs twice, one time described by its name (overt) and one time described as placebo (covert). Wash-out periods and blood sampling frames were drug-specific (Table 1) and extended to $>7$ and $\geq 5$ expected drug plasma half-life, respectively. Expected plasma half-life was about $1 \mathrm{~h}$ for cephalexin [24, 25], $2 \mathrm{~h}$ for ibuprofen [26], and $2.3 \mathrm{~h}$ for paracetamol [27].

\section{Participants}

We enrolled healthy (based on medical history, complete blood count, renal profile, and liver profile within 30 days), non-pregnant, non-lactating adults (age 18-60 years) with a body mass index (BMI) $\leq 35 \mathrm{~kg} / \mathrm{m}^{2}$, who accepted to abstain from taking any medication (including over-thecounter) for 1 week and from smoking, alcohol, and 
Table 1 Main characteristics of three randomized, 2-period, 2-sequence, cross-over studies comparing three drugs described by their name or as placebo

\begin{tabular}{llll}
\hline Drug name (dose) & Cephalexin (500 mg) & Ibuprofen (400 mg) & Paracetamol (500 mg) \\
\hline Participants, No. (sex) & $41(\mathrm{M}), 9(\mathrm{~F})$ & $27(\mathrm{M}), 3(\mathrm{~F})$ & $47(\mathrm{M}), 3(\mathrm{~F})$ \\
Age, mean (SD), year & $30.8(6.2)$ & $31.4(6.6)$ & $31.2(5.4)$ \\
BMl, mean (SD), $\mathrm{kg} / \mathrm{m}^{2}$ & $24.8(3.1)$ & $25.0(4.2)$ & $25.2(3.1)$ \\
Adverse events (No.) & Near fainting (1) & None & Localized rash (1) \\
Washout period, hour & 24 & 24 & 48 \\
Sampling frame, hour & 6 & 10 & 14 \\
Assay range, $\mu \mathrm{g} / \mathrm{ml}$ & $0.50-120$ & $0.25-60$ & $0.10-40$
\end{tabular}

${ }^{\mathrm{a}}$ All adverse events were mild and resolved spontaneously

caffeine for $48 \mathrm{~h}$ before and throughout each study periods. Subjects with a history of hypersensitivity to the drug to be used or with recent (one week) acute illness were excluded. For menstruating women, the study was conducted 5 to 19 days after the last menstrual period and after obtaining a negative urine pregnancy test.

The study was conducted at the King Faisal Specialist Hospital \& Research Center (KFSH\&RC), Riyadh from February 2012 through February 2013 after obtaining approval of the KFSH\&RC Research Ethics Committee. Volunteers were compensated based on the Wage-Payment model [28] in a prorated manner. The study followed published ethical guidelines on deception use in clinical research [29-32]. A written "consent" (specific to the drug to be administered) was obtained from each volunteer; being told that the study compares the effects of tablets/ capsules containing placebo to those containing the particular drug on a new serum marker, that it aims to determine how much of the observed changes in the serum marker is not related to the particular drug, and that they will each receive both the drug and placebo in a random sequence. At the completion of the three studies and after obtaining their monetary compensation, the volunteers were contacted for debriefing on the actual study aim and design and for delayed full consenting.

\section{Procedures and interventions}

The three drugs were purchased from retail pharmacies in Riyadh, Saudi Arabia. Brand name, manufacturer name, batch number, manufacturing date, and expiry date were: Keflex 500 mg, Facta Italy, 000301, 12/2010, and 12/2013 for cephalexin; Ibuprofen $400 \mathrm{mg}$, Hamol Ltd. UK, 1EE, 5/2010, and 5/2015 for ibuprofen; and Panadol 500 mg, GlaxoSmithKline, 110,216, 2/2011, and $2 / 2015$ for paracetamol.

Few days before the study, the volunteers were reminded to abstain from smoking, alcohol, and caffeine for $48 \mathrm{~h}$, from food for $10 \mathrm{~h}$, and from water for one hour, and to have $\geq 7 \mathrm{~h}$ of good sleep before each study period. Compliance with study instructions was checked before administering the drugs. The drugs were administered (by $\mathrm{MMH}$ ) with $240 \mathrm{ml}$ of water at room temperature. To enhance the placebo effect, immediately before drug administration, the volunteers were individually briefed and requested to read and sign an additional "consent" document that stated: "As you know, we are doing this study to determine how much of the change in serum marker level that occurs after ingestion of (name and dose of drug) is not related to (name of drug) effect but to placebo effect. This study has two parts. One time you will take (name of drug) and one time you will take a placebo. The placebo is not known to affect the level of the marker. Today you are assigned to take (name of drug or placebo)." Overt drugs were dispensed from the original brand manufacturer bottle, whereas covert drugs were dispensed from a bottle labelled "placebo". Fasting from food and beverages continued for $4 \mathrm{~h}$ post-dosing. However, volunteers were allowed $120 \mathrm{ml}$ water per hour, starting one hour after drug administration. A standardized breakfast and dinner were provided $4 \mathrm{~h}$ and about $10 \mathrm{~h}$ after drug administration. Meal plans were identical in all parts of the studies. Strenuous physical activity was not permitted during study periods. Volunteers remained ambulatory or seated upright (unless deemed medically necessary) for the first four hours after drug administration and were under continuous observation regarding occurrence of adverse events and compliance with study procedures. In addition, they were directly asked about experiencing any adverse events at the time of last blood collection of each period and at the beginning of period- 2 .

Eighteen blood samples were obtained before and, at $0.16,0.33,0.5,0.66,0.83,1.0,1.25,1.5,1.75,2.0,2.5,3.0$, $3.5,4.0,4.5,5.0$, and $6 \mathrm{~h}$ after cephalexin administration, at $0.25,0.5,0.75,1.0,1.25,1.5,1.75,2.0,2.25,2.50,3.0$, $3.5,4.0,5.0,6.0,8.0$, and $10 \mathrm{~h}$ after ibuprofen administration, and at $0.25,0.5,0.75,1.0,1.25,1.5,1.75,2.0,2.5$, 3.0, 3.5, 4.0, 6.0, 8.0, 10, 12, and $14 \mathrm{~h}$ after paracetamol administration. Blood samples were collected in vacutainer tubes, centrifuged (3000 rpm for $10 \mathrm{~min}$ ) at room temperature within $15 \mathrm{~min}$, and plasma samples were 
harvested in clean polypropylene tubes and placed immediately at $-80{ }^{\circ} \mathrm{C}$.

Drug concentrations were blindly measured by in-house, locally-validated, reversed-phase high performance liquid chromatography (HPLC) assays. [33-35] Limits of quantifications are shown in Table 1. Intra-assay coefficient of variation $(\mathrm{CV}$, standard deviation/mean $\times 100)$ and bias (measured concentration/nominal concentration $\times 100$ ) were $\leq 3.1 \%$ and $\leq 5.0 \%$ for cephalexin, $\leq 3.8 \%$ and $\leq 7.0 \%$ for ibuprofen, and $\leq 11.6 \%$ and $\leq 14.0 \%$ for paracetamol. A typical assay run included a series of 10 standard samples (calibrators), several sets of four quality control samples (concentrations at 1 and 3 times lower quantitation limit and 0.5 and $0.8-0.9$ upper quantitation limit), and unknown samples. Standards and quality control samples were distributed throughout the unknown samples. Samples from the two periods of each volunteer were analyzed in the same assay run. Samples with drug concentrations greater than the upper quantitation limit were re-assayed after dilution. Drug concentrations below the lower quantitation limit were assigned zero value. Drug concentrations of missing samples were assigned the value of the average concentration of the two flanking samples in the same period.

\section{Randomization}

Three randomization schedules (one for each drug) were generated (by $\mathrm{MMH}$ ) using an online program [36]. For each study, volunteers were block-randomized (block size $=2$ ) to one of two sequences (drug described by its name followed by drug described as placebo and vice versa). Assignment was concealed from recruiting study coordinators and potential participants.

\section{Deception and blinding}

Study coordinators and volunteers were deceived regarding study aim and design. Volunteers were deceived in the period when they were given the drug described as placebo. To enhance deception, volunteers were requested not to reveal their assignments to the coordinators. Drug concentrations and pharmacokinetic analysis were performed blinded to assignment.

\section{Sample size}

Calculation of the sample size for each of the three studies was based on the primary analysis of a difference in drug half-life of $10 \%$, type I error of 0.05 , type II error of 0.1 , and about $10 \%$ withdrawal/dropout rate. We estimated that mean and standard deviation (SD) drug half-life are, respectively, 1.0 and $0.21 \mathrm{~h}$ for cephalexin [24, 25], 2.0 and $0.30 \mathrm{~h}$ for ibuprofen [26], and 2.3 and $0.46 \mathrm{~h}$ for paracetamol [27]. The calculated sample size, allowing for withdrawals/dropouts, was 50 for the cephalexin study, 30 for the ibuprofen study, and 50 for the paracetamol study.

\section{Outcome measures and analysis}

The following pharmacokinetic parameters were determined using standard non-compartmental method: $\mathrm{AUC}_{\mathrm{T}}$ calculated by linear trapezoidal method, terminal rate constant $(\lambda)$ calculated by linear least-squares regression analysis from a plot of natural log-transformed plasma concentration versus time curve, $\mathrm{AUC}_{\mathrm{I}}$ calculated as the sum of $\mathrm{AUC}_{\mathrm{T}}$ plus the ratio of last quantifiable plasma level/ $\mathrm{\lambda}, \mathrm{AUC}_{\mathrm{T}} / \mathrm{AUC}_{\mathrm{I}}, \mathrm{C}_{\max }$ determined directly from the observed data, $\mathrm{C}_{\max } / \mathrm{AUC}_{\mathrm{I}}, \mathrm{T}_{\max }$ determined directly from the observed data, $t_{1 / 2}$ calculated as natural $\log$ of $2 / \lambda$, , and AUC to $\mathrm{T}_{\max }$ of the overt drug $\left(\mathrm{AUC}_{\text {Overttmax }}\right)$ calculated by linear trapezoidal method.

The primary outcome measure was $t_{1 / 2}$. Secondary outcome measures were the other pharmacokinetic parameters. Outcome measures were evaluated by analysis of variance (ANOVA) after natural log-transformation, except for $\mathrm{T}_{\max }$. The model included sequence, subjects nested within sequence, period, and intervention (covert vs overt drug administration). Mean square residual error (MSR) was used to test the significance of period and intervention effects. Subjects nested in sequence mean square was used to test the significance of sequence effect. Secondary analysis evaluated covert-overt drug ABE: the $90 \% \mathrm{CI}$ on the difference between means of log-transformed values was determined (using MSR) and the antilog of the $90 \%$ CI limits were compared to the $80.00 \%$ and $125.00 \%$ bioequivalence limits. The null hypothesis of no placebo effect on drug bioavailability was rejected if the covert-overt drug difference was not significant at the 0.05 level. The null hypothesis of lack of bioequivalence was rejected if the $90 \% \mathrm{CI}$ was entirely within the $80.00 \%$ to $125.00 \%$ limits. We also calculated the percentage of individual covert/overt pharmacokinetic ratios that were $<75 \%$ or $>125 \%$ and their mean deviation from $100 \%$.

Pharmacokinetic calculations and statistical analysis were performed (by $\mathrm{MMH}$ ) on a personal computer using Microsoft Excel (Version 2010) with relevant add-ins (PK Functions for Microsoft Excel, JI Usansky, A Desai, and D Tang-liu, Department of pharmacokinetics and Drug Metabolism, Allergan Irvine, CA, USA) and IBM SPSS Statistics version 21 software (SPSS Inc., Chicago, Ill, USA), respectively. Analyses were not adjusted for multiple comparisons. Two-tailed $p$ values are reported.

\section{Results}

A total of 130 (Fig. 1) healthy volunteers participated in three two-period, two-sequence, cross-over studies that compared two single oral doses of cephalexin, 


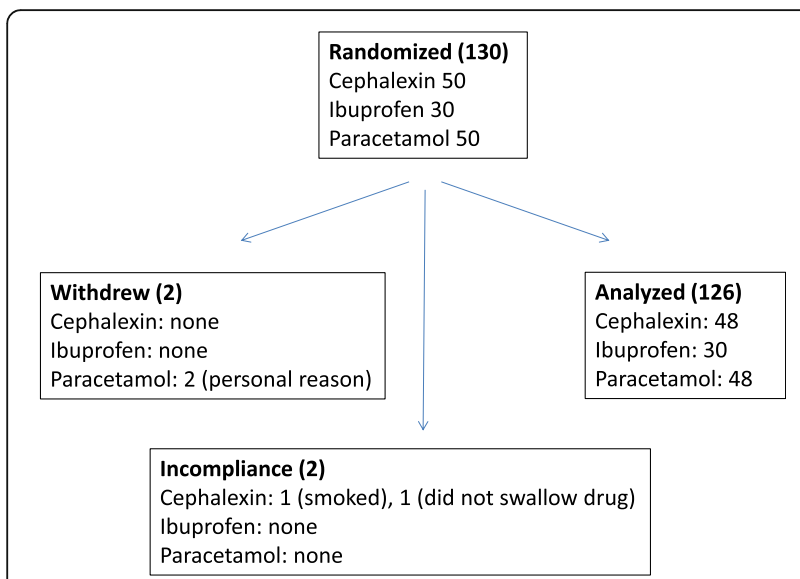

Fig. 1 Flow of participants through the study

ibuprofen, or paracetamol, each described by its name (overt) or as placebo (covert). As shown in Table 1, $6-18 \%$ of the volunteers per study were females. Mean (SD) age ranged from 30.8 (6.2) to 31.4 (6.6) years and mean BMI from 24.8 (3.1) to 25.2 (3.1) kg/ $\mathrm{m}^{2}$. As shown in Figure 1, withdrawal rate ranged from $0 \%$ (ibuprofen) to $4 \%$ (cephalexin and paraceta$\mathrm{mol}$ ). Withdrawal reasons were personal (one volunteer withdrew before period-1 and one after period-2, refusing to provide post-study consent) or incompliance with study procedures (one volunteer smoked during the study and one did not swallow cephalexin tablet). We were able to contact 68 (54\%) out of the 127 volunteers who completed the study for poststudy debriefing and consenting, all gave full informed consent except one volunteer (noted above), whose data were removed from analysis. Adverse events occurred in $0 \%$ (ibuprofen) to $2 \%$ (cephalexin and paracetamol) of volunteers (Table 1); all were minor and resolved spontaneously.

Eighteen blood samples were obtained over 6 to $14 \mathrm{~h}$ in each study period with a washout interval ranging from 24 to $48 \mathrm{~h}$ (Table 1). There were no missing blood samples or interfering plasma peaks in any of the 3 drug assays. Baseline plasma concentrations were below the assay detection limits in all volunteers. In one volunteer in the cephalexin study, cephalexin concentration was not measurable in any sample during one study period (the volunteer, note above, admitted that he did not swallow the cephalexin tablet). The entire data of this volunteer and of the volunteer who did not provide post-study consent were not included in further analysis. Mean untransformed and natural logarithm-transformed concentration-time curve of the three drugs when administered overtly or covertly are presented in Fig. 2 and Fig. 3, respectively. The results are consistent with the results of previous studies on cephalexin [24, 25], ibuprofen [26], and paracetamol [27].

We were able to calculated $\lambda$ in all analyzed periods. No outlier values for $\mathrm{AUC}_{\mathrm{T}}, \mathrm{AUC}_{\mathrm{I}}$, or $\mathrm{C}_{\max }$ were identified/removed from analysis. Main pharmacokinetic parameters of the three drugs when administered overtly or covertly are summarized in Table $2 . \mathrm{AUC}_{\mathrm{T}} / \mathrm{AUC}_{\mathrm{I}}$ ranged from 93\% (ibuprofen) to $97 \%$ (cephalexin and paracetamol), indicating adequate sampling frames. MSR from ANOVA analysis and calculated intra-subject CV for each of the 3 drugs are presented in Table 3. The intra-subject CV ranged from 5.5\% (paracetamol) to 9.5\% (cephalexin) for $\mathrm{AUC}_{\mathrm{I}}$ and from $23.1 \%$ (paracetamol) to $29.8 \%$ (ibuprofen) for $C_{\max }$. There were no significant $(p>0.05)$ period or sequence effects in any of the three studies.

\section{No significant change in the pharmacokinetics of three drugs when described as placebo}

$P$ values from ANOVA comparing the pharmacokinetic parameters for the three drugs when each was administered overtly or covertly are presented in Table 2 . There were no significant differences between the two conditions except for ibuprofen $\mathrm{AUC}_{\text {Overttmax }}(P=0.04)$. In particular, the $p$ value for $\log$ transformed $\lambda$ for the three drugs ranged from 0.052 for ibuprofen to 0.99 for cephalexin.

\section{Average bioequivalence of three drugs when described by their names or as placebo}

Table 3 summarizes the results of bioequivalent analysis comparing $\mathrm{AUC}_{\mathrm{T}}, \mathrm{AUC}_{\mathrm{I}}$, and $\mathrm{C}_{\max }$ of the three drugs when they were described by their names compared to when they were described as placebo. There was little difference between the two conditions. Absolute deviation of point estimates from $100 \%$ was $\leq 3.34, \leq 1.46$, and $\leq 12.89$ percentage points for $\mathrm{AUC}_{\mathrm{T}}, \mathrm{AUC}_{\mathrm{I}}$, and $\mathrm{C}_{\text {max }}$, respectively. Further, none of the $\mathrm{AUC}_{\mathrm{T}}$ or $\mathrm{AUC}_{\mathrm{I}}$ 90\% CIs failed to fall within the $80.00-125.00 \%$ bioequivalence limits and only one of the three $C_{\max } 90 \%$ CIs barely failed to do so (76.66-98.99 for ibuprofen). The results are also depicted in Fig. 4. Power analysis revealed that the three studies had a power of $>0.9$ to show average bioequivalence for $\mathrm{AUC}_{\mathrm{T}}, \mathrm{AUC}_{\mathrm{I}}, \mathrm{C}_{\max }$, and $\mathrm{C}_{\max /} \mathrm{AUC} \mathrm{C}_{\mathrm{I}}$, except for ibuprofen $\mathrm{C}_{\max }$ and $\mathrm{C}_{\max } / \mathrm{AUC}_{\mathrm{I}}$, were the power was 0.32 and 0.44 , respectively.

Table 3 and Fig. 4 also present point estimates and $90 \%$ CIs of $A U C_{\text {Overtmax }}$ and $C_{\max } / A C_{I}$ of the three drugs under the overt and covert conditions. Only ibuprofen $\mathrm{AUC}_{\text {Overtmax }}$ and $\mathrm{C}_{\max } / \mathrm{AUC}_{\mathrm{I}}$ and paracetamol $\mathrm{AUC}_{\text {Overttmax }} 90 \% \mathrm{CIs}$ failed to show bioequivalence and none showed bioinequivalence. 

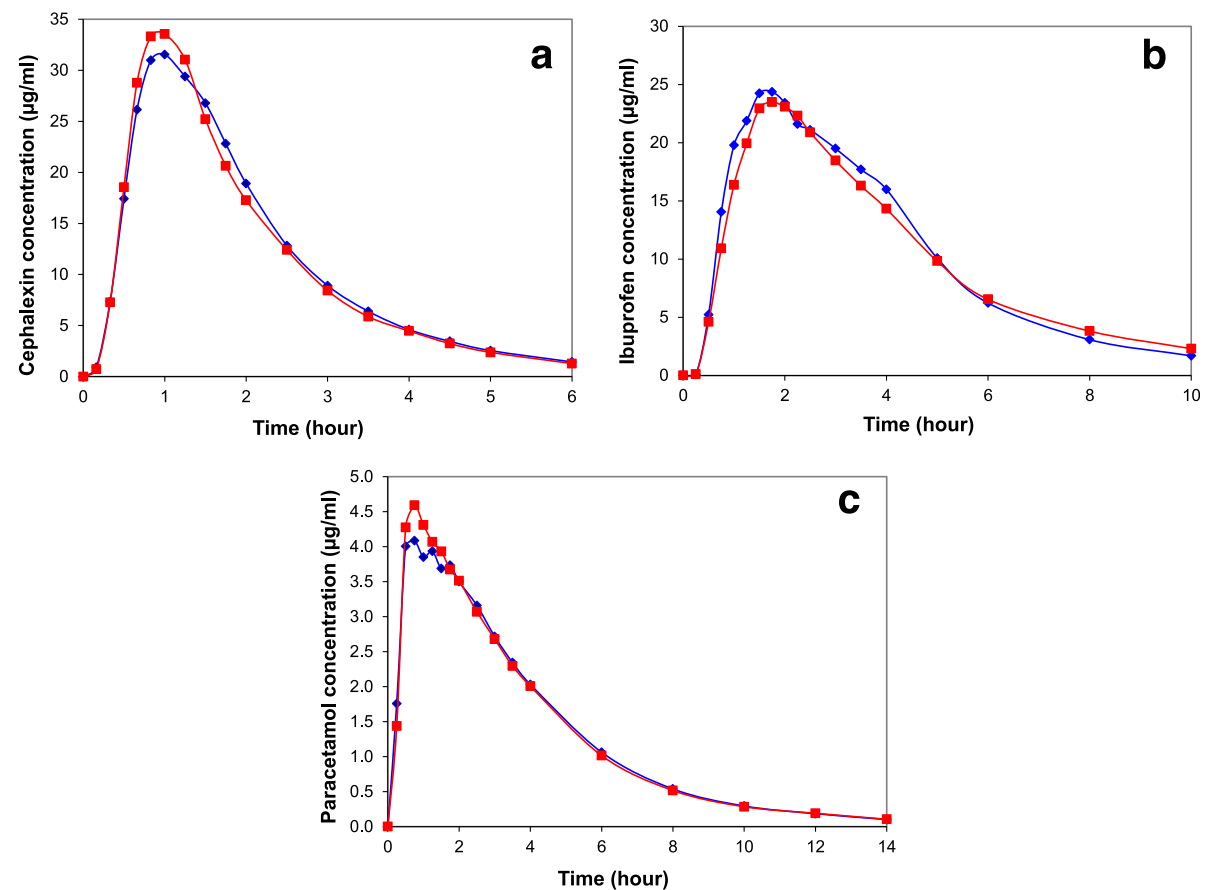

Fig. 2 Time-concentration curves of cephalexin (a), ibuprofen (b), and paracetamol (c) described as such (blue diamonds) or as placebo (red squares). Data represent mean concentrations
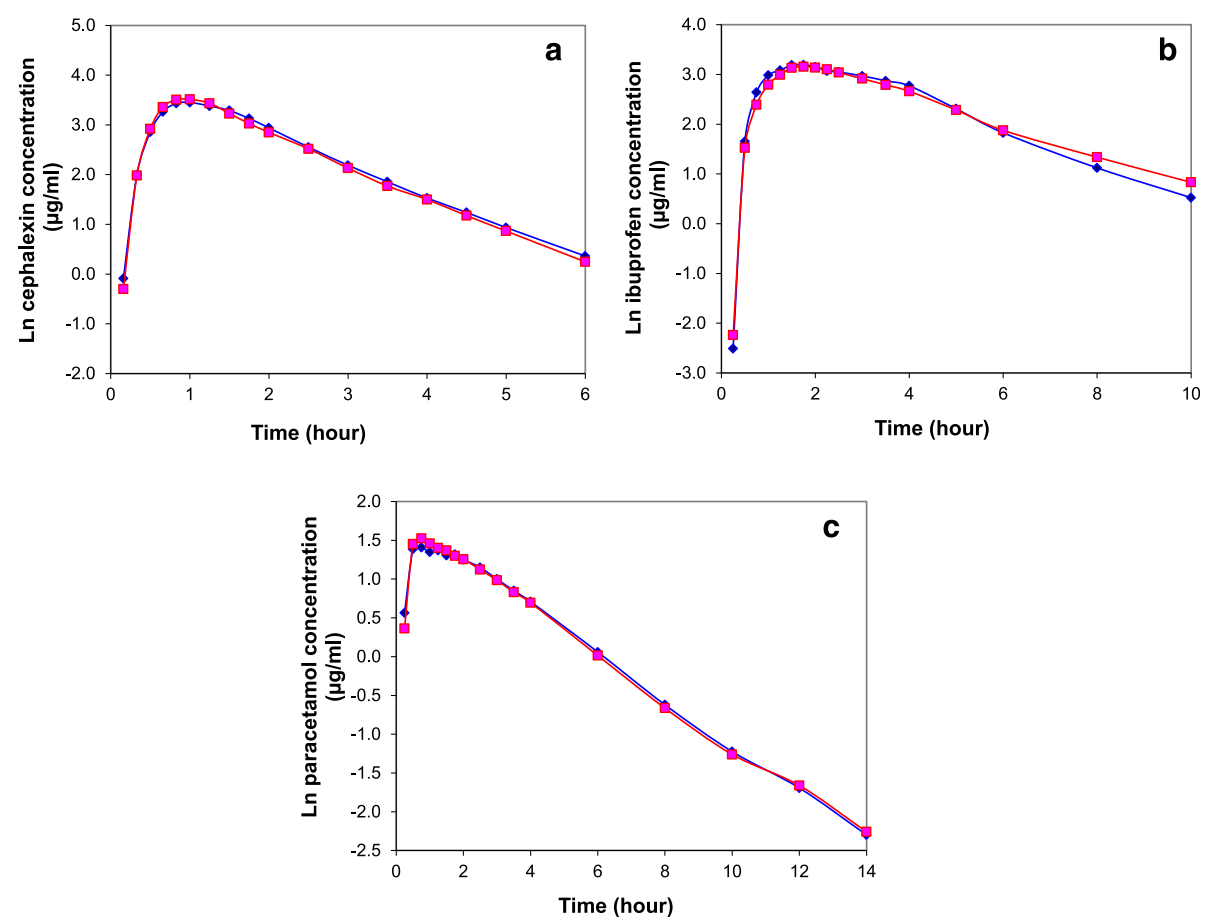

Fig. 3 Time-log-concentration curves of cephalexin (a), ibuprofen (b), and paracetamol (c) described as such (blue diamonds) or as placebo (red squares). Data represent mean natural log-transformed concentrations 
Table 2 Main pharmacokinetic parameters of three drugs described by their name (overt) or as placebo (covert)

\begin{tabular}{|c|c|c|c|c|c|c|c|c|c|}
\hline \multirow[b]{2}{*}{ Parameter } & \multicolumn{3}{|l|}{ Cephalexin } & \multicolumn{3}{|l|}{ Ibuprofen } & \multicolumn{3}{|l|}{ Paracetamol } \\
\hline & Overt & Covert & $P$ value & Overt & Covert & $P$ value & Overt & Covert & $P$ value \\
\hline$A \cup C_{T}(\mu g . h r . / m l)$ & $68.53 \pm 26.39$ & $67.20 \pm 24.96$ & 0.47 & $104.38 \pm 20.08$ & $101.43 \pm 21.82$ & 0.27 & $18.48 \pm 3.98$ & $18.52 \pm 3.68$ & 0.62 \\
\hline $\mathrm{A} \cup C_{\mathrm{I}}(\mu \mathrm{g} \cdot \mathrm{hr} . / \mathrm{ml})$ & $71.11 \pm 28.04$ & $69.61 \pm 25.77$ & 0.46 & $109.40 \pm 20.45$ & $109.65 \pm 21.54$ & 0.99 & $19.04 \pm 4.11$ & $19.07 \pm 3.79$ & 0.65 \\
\hline$C_{\max }(\mu \mathrm{g} / \mathrm{ml})$ & $40.16 \pm 16.51$ & $39.32 \pm 17.01$ & 0.68 & $31.97 \pm 6.53$ & $29.17 \pm 10.34$ & 0.08 & $5.72 \pm 1.74$ & $6.08 \pm 1.62$ & 0.16 \\
\hline $\mathrm{T}_{\max }(\mathrm{hr})$ & $1.11 \pm 0.42$ & $1.09 \pm 0.49$ & 0.82 & $1.86 \pm 0.90$ & $2.02 \pm 1.21$ & 0.58 & $1.01 \pm 0.66$ & $0.94 \pm 0.62$ & 0.45 \\
\hline$\lambda\left(h r^{-1}\right)$ & $0.68 \pm 0.15$ & $0.67 \pm 0.13$ & 0.99 & $0.39 \pm 0.06$ & $0.35 \pm 0.08$ & 0.052 & $0.30 \pm 0.05$ & $0.30 \pm 0.05$ & 0.51 \\
\hline$t_{1 / 2}(h r)$ & $1.07 \pm 0.23$ & $1.07 \pm 0.23$ & 0.99 & $1.85 \pm 0.53$ & $2.11 \pm 0.74$ & 0.052 & $2.33 \pm 0.35$ & $2.35 \pm 0.35$ & 0.51 \\
\hline$C_{\max } / A \cup C_{1}\left(h r^{-1}\right)$ & $0.57 \pm 0.12$ & $0.56 \pm 0.12$ & 0.86 & $0.30 \pm 0.05$ & $0.27 \pm 0.08$ & 0.06 & $0.30 \pm 0.07$ & $0.33 \pm 0.10$ & 0.14 \\
\hline$A \cup C_{T} / A \cup C_{1}$ & $0.97 \pm 0.02$ & $0.97 \pm 0.02$ & 0.84 & $0.95 \pm 0.05$ & $0.93 \pm 0.08$ & 0.10 & $0.97 \pm 0.01$ & $0.97 \pm 0.02$ & 0.74 \\
\hline$A \cup C_{\text {Overttmax }}(\mu \mathrm{g} . \mathrm{hr} . / \mathrm{ml})$ & $17.87 \pm 10.91$ & $19.98 \pm 15.56$ & 0.73 & $22.65 \pm 9.84$ & $24.69 \pm 25.77$ & 0.04 & $2.51 \pm 2.11$ & $3.02 \pm 2.90$ & 0.10 \\
\hline
\end{tabular}

Data are unadjusted mean $\pm \mathrm{SD}$ of untransformed values. $A \cup C_{T}$ is area-under-the-plasma-concentration-time curve from time 0 to last measured concentration. $A U C_{1}$ is area-under-the-plasma-concentration-time curve extrapolated to infinity. $C_{\max }$ and $T_{\max }$ are first measured maximum plasma level and its time, respectively. $\lambda$ is terminal elimination constant. $t_{1 / 2}$ is plasma half-life. $A U C_{\text {Overtmax }}$ is area-under-the-plasma-concentration-time curve to $T_{\max }$ under overt drug administration.

$P$ values were obtained from Analysis of variance (ANOVA) of natural logarithm-transformed values except for $T_{\max }$.

Individual bioequivalence of three drugs when described by their names or as placebo

The percentages of individual covert/overt $\mathrm{AUC}_{\mathrm{T}}$, $\mathrm{AUC}_{\mathrm{I}}, \mathrm{C}_{\max }, \mathrm{AUC}_{\text {Overttmax, }}$ and $\mathrm{T}_{\max }$ ratios that are less than 0.75 or more than 1.25 are presented in Fig. 5.
About 6.9\% (least square mean) of the 126 individual ratios were outside the range of 0.75 to 0.125 for $\mathrm{AUC}_{\mathrm{T}}$ with a range of $2.0 \%$ (paracetamol) to $16.7 \%$ (ibuprofen), $3.2 \%$ for $\mathrm{AUC}_{\mathrm{I}}$ with a range of $2.0 \%$ (paracetamol) to $4.2 \%$ (cephalexin), $34.4 \%$ for $C_{\max }$ with a range from $25.0 \%$ (cephalexin) to $44.9 \%$ (paracetamol), $71.6 \%$ for

Table 3 Bioequivalence comparison of three drugs described by their name (overt) or as placebo (covert)

\begin{tabular}{|c|c|c|c|}
\hline Drug name & Cephalexin & Ibuprofen & Paracetamol \\
\hline \multicolumn{4}{|l|}{$A \cup C_{T}$} \\
\hline $\mathrm{PE}(\mathrm{Cl})$ & $98.47 \%$ (95.04-102.02) & 96.66\% (91.79-101.79) & $100.62 \%(98.77-102.50)$ \\
\hline MSR & 0.011 & 0.014 & 0.003 \\
\hline$C V$ & $10.5 \%$ & $11.9 \%$ & $5.5 \%$ \\
\hline \multicolumn{4}{|l|}{$A \cup C_{1}$} \\
\hline $\mathrm{PE}(\mathrm{Cl})$ & 98.54\% (95.34-101.83) & 99.96\% (96.30-103.76) & $100.56 \%(98.71-102.45)$ \\
\hline MSR & 0.009 & 0.007 & 0.003 \\
\hline CV & $9.5 \%$ & $8.4 \%$ & $5.5 \%$ \\
\hline \multicolumn{4}{|l|}{$C_{\max }$} \\
\hline $\mathrm{PE}(\mathrm{Cl})$ & $97.87 \%$ (89.81-106.65) & $87.11 \%$ (76.66-98.99) & 106.79\% (98.84-115.37) \\
\hline MSR & 0.063 & 0.085 & 0.052 \\
\hline CV & $25.5 \%$ & $29.8 \%$ & $23.1 \%$ \\
\hline \multicolumn{4}{|l|}{ AUC Overttmax } \\
\hline $\mathrm{PE}(\mathrm{Cl})$ & $96.64 \%$ (82.17-113.66) & 64.44\% (45.32-91.62) & $71.36 \%$ (51.45-98.96) \\
\hline MSR & 0.224 & 0.642 & 0.931 \\
\hline CV & $50.1 \%$ & $94.9 \%$ & $124.0 \%$ \\
\hline \multicolumn{4}{|l|}{$C_{\max } / A \cup C_{1}$} \\
\hline $\mathrm{PE}(\mathrm{Cl})$ & $99.32 \%$ (93.00-106.08) & 87.15\% (77.19-98.39) & $106.19 \%(99.15-113.73)$ \\
\hline MSR & 0.037 & 0.076 & 0.041 \\
\hline CV & $19.4 \%$ & $28.1 \%$ & $20.5 \%$ \\
\hline
\end{tabular}

$\mathrm{AUC}_{\mathrm{T}}$ is area-under-the-plasma-concentration-time curve from time 0 to last measured concentration. $\mathrm{AUC}_{\mathrm{I}}$ is area-under-the-plasma-concentration-time curve extrapolated to infinity. $C_{\max }$ is first measured maximum plasma level. $A U C_{\text {Overtmax }}$ is area-under-the-plasma-concentration-time curve to $T_{\max }$ under overt drug administration. PE is point estimate (antilog of the difference between means of log-transformed data). $\mathrm{Cl}$ is parametric $90 \%$ confidence interval on PE. MSR is mean square residual from analysis of variance. $\mathrm{CV}$ is intra-subject coefficient of variation, calculated as $100 \times(\exp (\mathrm{MSR})-1)^{0.5}$. The number of subjects that were analyzed was 48 for cephalexin, 30 for ibuprofen, and 48 for paracetamol. 


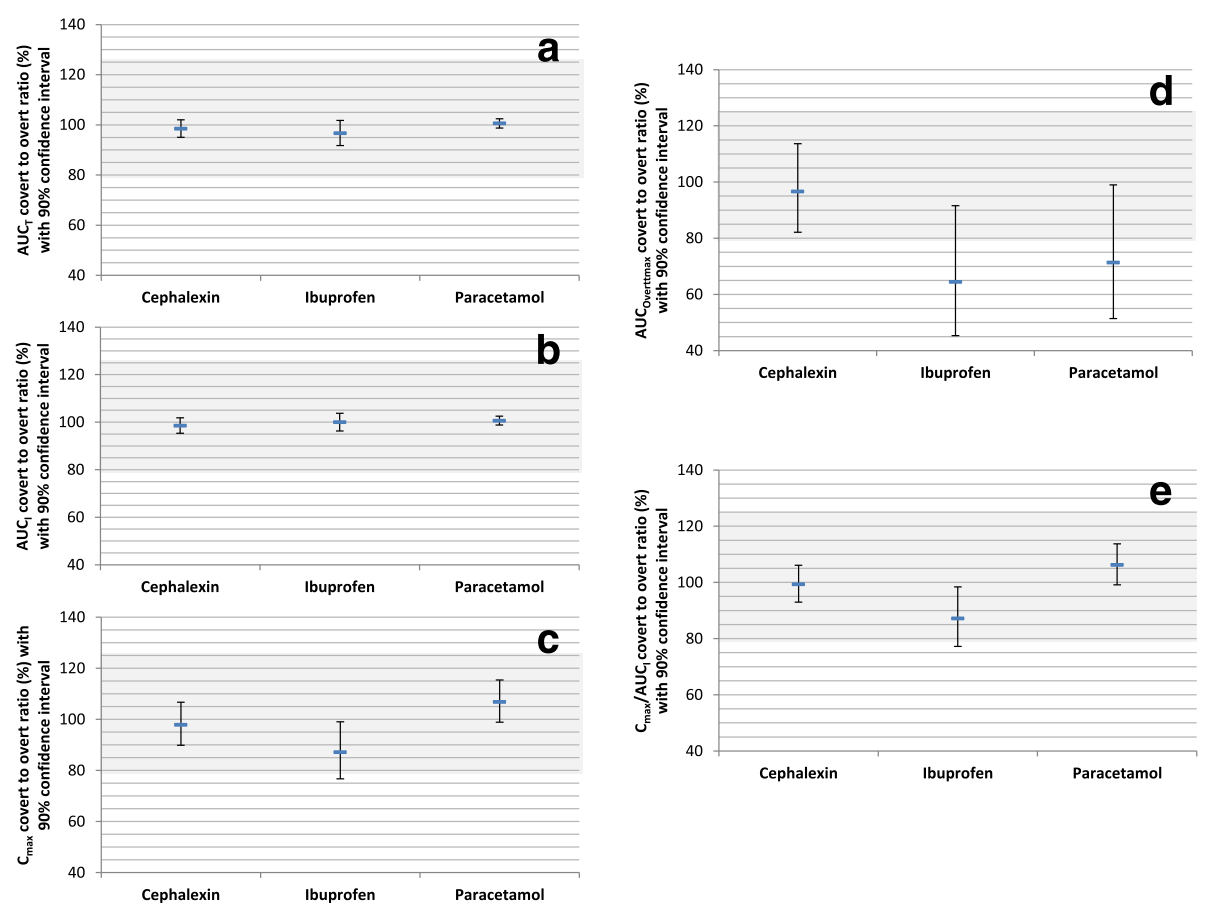

Fig. 4 Average bioequivalence evaluation of covert-overt cephalexin, ibuprofen, and paracetamol. Data represent point estimate (antilog of mean covert-overt difference of log-transformed values) and parametric $90 \%$ confidence interval. The shaded area indicates the area of bioequivalence $(80.00 \%$ to $125.00 \%)$. a, bioequivalence evaluation of area-under-the-concentration-time curve to last quantifiable concentration $\left(A \cup C_{T}\right)$. $\mathbf{b}$, bioequivalence evaluation of area-under-the-concentration-time curve extrapolated to infinity $\left(A \cup C_{1}\right)$. $\mathbf{c}$, bioequivalence evaluation of maximum concentration $\left(\boldsymbol{C}_{\text {max }}\right)$. $\mathbf{d}$, bioequivalence evaluation of area-under-the-concentration-time curve extrapolated to overt $T_{\max }\left(A \cup C_{\text {Overttmax }}\right.$. e, bioequivalence evaluation of $C_{\max } / A \cup C_{1}$

AUC $_{\text {Overttmax }}$ with a range of $67.3 \%$ (paracetamol) to $76.7 \%$ (ibuprofen), and $60.2 \%$ for $\mathrm{T}_{\max }$ with a range of $45.8 \%$ (cephalexin) to $71.4 \%$ (paracetamol).

\section{Discussion}

The aim of the study was to examine the hypothesis that the drug*placebo interaction effect involves modulation of drug pharmacokinetics, namely, the knowledge that one is ingesting a drug would alter the pharmacokinetic parameters of the drug. We measured the effect of describing a drug as placebo (covert drug) on the pharmacokinetics of cephalexin, ibuprofen, and paracetamol. The three drugs were selected in part because of their known safety profiles. Ibuprofen and paracetamol were studied because of their expected familiarity (and hence ability to elicit a placebo effect) and cephalexin was used as "negative control" because of its expected unfamiliarity to study volunteers. We designed a two-period, two-sequence, cross-over study for each of the three drugs, with a $90 \%$ power to detect a $10 \%$ difference in $t_{1 / 2}$ between overtly and covertly administered drugs. Drug concentrations were blindly determined using in-house HPLC assays and $\mathrm{AUC}_{\mathrm{T}}$, $\mathrm{AUC}_{\mathrm{I}}, \mathrm{C}_{\max }, \mathrm{T}_{\max }, \lambda, \mathrm{t} 1 / 2$, and $\mathrm{AUC}_{\text {Overttmax }}$ were blindly estimated by the standard non-compartmental method. We compared the pharmacokinetic parameters under the two administration conditions using ANOVA, computed 90\% CIs on the difference (covert-overt) between the means of their log-transformed values and compared them to the standard bioequivalence range of $80.00 \%$ to $125.00 \%$, and calculated the percentages of the untransformed individual pharmacokinetic covert/overt ratios that are outside the $+25 \%$ range. We found that: 1 ) there is no placebo effect on any of the pharmacokinetic parameters studied, 2) the two conditions of administering the drugs resulted in bioequivalent profiles, and 3) about 34.4\%, $71.6 \%$, and $60.2 \%$ of individual covert/overt ratios for $\mathrm{C}_{\text {max }}, \mathrm{AUC}_{\text {Overttmax }}$, and $\mathrm{T}_{\max }$, respectively, were outside the $\pm 25 \%$ range.

\section{Describing drugs as placebos doesn't significantly alter their pharmacokinetic parameters}

We found no significant differences between the covert and overt conditions in any of the studied pharmacokinetic parameters of the three drugs. This is in contrast to the results of a previous, similarly designed cross-over study on $300 \mathrm{mg}$ caffeine [7], which found that mean plasma caffeine levels were consistently lower in the terminal part of the 14-h concentration-time curve, mean caffeine AUC was significantly lower, and mean plasma caffeine terminal half-life was significantly shorter when 


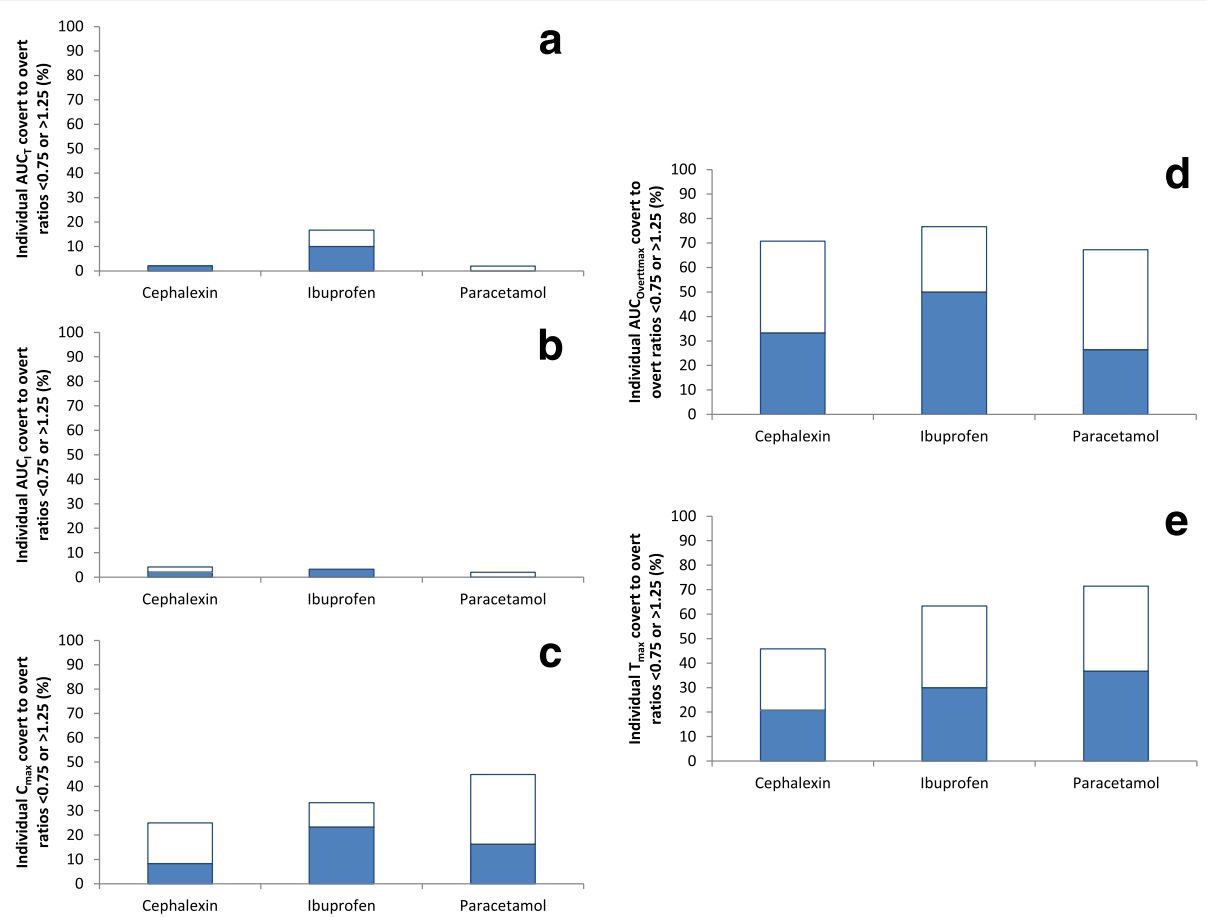

Fig. 5 Individual bioequivalence evaluation of covert-overt cephalexin, ibuprofen, and paracetamol. Data represent percentage of individual ratios that are 0.75 (closed bars) or ${ }^{>} 1.25$ (open bars). a, bioequivalence evaluation of area-under-the-concentration-time curve to last quantifiable concentration $\left(A \cup C_{T}\right)$. $\mathbf{b}$, bioequivalence evaluation of area-under-the-concentration-time curve extrapolated to infinity $\left(A \cup C_{1}\right)$. $\mathbf{c}$, bioequivalence evaluation of maximum concentration $\left(C_{\max }\right) \cdot \mathbf{d}$, bioequivalence evaluation of area-under-the-concentration-time curve extrapolated to overt $T_{\max }$ $\left(A \cup C_{\text {Overttmax }}\right)$. e, bioequivalence evaluation of time to maximum concentration $\left(T_{\text {max }}\right)$

caffeine was given covertly. The reason for the discrepancy is not clear. It is possible that the results of the caffeine study were due to chance, especially because the study was exploratory, had a low power (22 subjects), and tested a novel mechanism, which are expected to increase the probability of false discovery, despite having statistically significant results $[37,38]$. However, it is also possible that a placebo effect for ibuprofen and paracetamol was not successfully elicited in the current study; other outcomes (for example pain reduction) unfortunately were not examined. There are several modulators of the placebo effect, including conditioning [39], expectancy, suggestion, personality, desire for symptom change, and affective state [40].

\section{Average bioequivalence of the three drugs under covert and overt conditions}

Since no placebo effect was observed on the pharmacokinetic parameters of the three drugs, the volunteers could be considered to have received the same drug product. We thus used the data to explore the extent of biovariability that can be observed when comparing a drug product to itself. We found that the two conditions of administering the drugs resulted in bioequivalent profiles; only one of the three $C_{\max } 90 \%$ CIs barely failed to show bioequivalence using the strict $80 \%-125 \%$ bioequivalence limits. The outcome of a cross-over bioequivalence study is affected by its sample size and intra-subject variability. In retrospect, power analysis revealed that the ibuprofen study has only a 0.32 power to show bioequivalence for $\mathrm{C}_{\max }$.

Intra-subject variability can be due to the drug substance itself (being readily affected by physiological variability of the volunteer), product quality variability, analytical variability, or unexplained random variation [41]. In a typical bioequivalence study comparing test and reference drug products, intra-subject variability includes, in addition, variability due to differences between the two products [41]. When a generic drug product is declared therapeutically equivalent to a reference product, it is expected that any difference between the two products should be no greater than the difference between two batches of the reference product. In fact, reviewing the bioequivalence studies of its approved generic products, the US Food and Drug Administration (FDA) found a mean generic-reference deviation of $3.47 \%$ for AUC and $4.29 \%$ for $C_{\max }$ in one study [42] and $3.56 \%$ for $\mathrm{AUC}$ and $4.35 \%$ for $\mathrm{C}_{\max }$ in another [43]. Commonly, there are several marketed drug products that are linked by a chain of reference [44]; concerns have been raised that reference-bioequivalent generic products may not be bioequivalent to each other. However, secondary analysis 
of 120 bioequivalence studies on three immunosuppressants and six selected drugs showed a mean generic-generic deviation of $4.5 \%$ for $\mathrm{AUC}_{\mathrm{T}}$ and $5.1 \%$ for $C_{\max }$ [45]. Interestingly, in the current study, we found that covert-overt deviation was $\leq 3.34, \leq 1.46$, and $\leq 12.89$ percentage points for $\mathrm{AUC}_{\mathrm{T}}, \mathrm{AUC}_{\mathrm{I}}$, and $C_{\max }$, respectively; suggesting that most of the deviation observed in typical bioequivalence studies is not related to using two different products.

Bioequivalence studies' guidelines of regulatory agencies (except for Health Canada (HC)) are silent regarding blinding of study volunteers [18-20]. Our results suggest that failure to blind would not be expected to have a negative impact; even describing the drugs as placebo did not affect their pharmacokinetic parameters.

There is disagreement among the different regulatory guidelines on the bioequivalence criteria for $\mathrm{C}_{\max }$, whereas some require that the $90 \% \mathrm{CI}$ should fall within the 80 $125 \%$ or the $70-143 \%$ limits, others require only the point estimate to fall within the $80-125 \%$ limits [18-20]. Our results suggest that requiring the $90 \% \mathrm{CI}$ to fall within the $80-125 \%$ limits may be too strict as ibuprofen reference product failed this criteria when compared to itself, despite having a relatively large sample size.

For drugs where time of onset of effect is important, the US FDA and $\mathrm{HC}$ recommend that the $90 \% \mathrm{CI}$ of AUC truncated at reference product median $\mathrm{T}_{\max }$ or AUC $_{\text {Reftmax }}$, respectively, should be within the $80-125 \%$ limits [18-20]. Our results indicate that such criteria would be difficult to achieve; only one of the three $90 \%$ CIs of AUC Overttmax $_{\text {was }}$ within the $80-125 \%$ limits.

\section{Individual bioequivalence of the three drugs under covert and overt conditions}

It has been argued that because $\mathrm{ABE}$ testing focuses on differences between mean values and relatively neglects differences between variances and subject-by-product interaction, it is possible that, despite establishing $\mathrm{ABE}$, a patient switched from a reference product to a generic product (or vice versa) could be over-dosed or underdosed and that some patients may have the highest drug exposure values with the reference product and lowest exposure values with the generic product and vice versa [46]. In fact, a bioequivalent study comparing generic and reference cyclosporine products found that $38 \%$ of individual $\mathrm{C}_{\max }$ ratios and $18 \%$ of individual AUC ratios were less than 0.80 despite having $90 \%$ CI within the 80-125\% limits [47].

Our findings that about $6.9 \%, 3.2 \%$, and $34.4 \%$ of individual covert/overt ratios for $\mathrm{AUC}_{\mathrm{T}}, \mathrm{AUC}_{\mathrm{I}}$, and $\mathrm{C}_{\max }$, respectively, were outside the $\pm 25 \%$ range indicate that most of the variation in individual ratios are not related to using different drug products but rather to the volunteers, drug moiety, study setting, drug assay, or random variations. Our findings are consistent with the results of previous studies [48, 49]. A simulation study (assuming inter-subject variability of $20 \%$ and intra-subject variability of $10 \%$ ) found that $11.1 \%$ of the reference/reference AUC ratios fell outside the $0.80-1.25$ range [48]. Further, in a fully-replicated bioequivalence study on the antiepileptic drug, lamotrigine, $3 \%$ and $18 \%$ of the generic/generic ratios and $3 \%$ and $9 \%$ of the reference/reference ratios for $\mathrm{AUC}$ and $\mathrm{C}_{\max }$, respectively, were outside the $0.75-1.25$ range [49].

Finally, it could be argued that some patients' distrust of reference-bioequivalent generic products $[12,13]$ could be related to generic products' different onset of action, which in turn may be related to different inactive ingredients or manufacturing processes. However, this is not likely. Onset of a drug effect is for the most part related to its pharmacodynamic characteristics and as shown in the current study, large variations in $\mathrm{T}_{\max }$ and AUC $_{\text {Overttmax }}(60.2 \%$ and $71.6 \%$ of individual ratios, respectively, were outside the $\pm 25 \%$ range) can be observed when comparing a product to itself.

\section{Limitations}

The interpretation of the results of this study is limited by the following. 1) Intervention's administration by an undeceived investigator may have reduced the placebo effect. 2) It is possible that the study setting and the drugs used were not conducive to elicit adequate placebo effect; thus the finding of no difference in pharmacokinetic parameters between the overt and covert conditions may not be related to the placebo effect not modulating drug bioavailability but rather to failure to induce a placebo effect. 3) The protocol-defined aim of the study was to examine if the drug*placebo effect involves modulating drug bioavailability; thus the findings regarding bioequivalence of drug products with themselves are based on post hoc analysis. 4) Our study was not designed to partition intra-subject variability into its various components. Thus, it is not clear how much of the observed variability is due to the drug products themselves (i.e. to drug product quality variability rather than to the drug moiety, random error, etc.).

\section{Conclusions}

This study couldn't confirm that awareness of drug ingestion can modulate its bioavailability. Although this may be due to inability to elicit adequate placebo effect, the results cast doubt on the concept that the drug*placebo interaction effect may involve modulating drug pharmacokinetics through mechanisms such as altering gastric emptying, intestinal transit time, or drug elimination. On the other hand, the study demonstrates that most of the generic-reference deviation observed in typical bioequivalence studies may not be related to using 
different products but is inherent in study design and setting, that failure to blind subjects in bioequivalence studies may not negatively impact validity of the results, that the $80-125 \%$ bioequivalence limits for $C_{\max }$ and $\mathrm{AUC}_{\text {Overttmax }} 90 \% \mathrm{CI}$ may be too strict, and that considerable intra-subject variability in $\mathrm{C}_{\max }, \mathrm{T}_{\max }$, and $\mathrm{AUC}_{\text {Reftmax }}$ would be expected even when comparing a drug product to itself.

\section{Abbreviations}

ABE: Average bioequivalence; ANOVA: Analysis of variance; $A \cup C_{T}$ : Areaunder-the-concentration-time-curve to last measured concentration; $A \cup C_{1}$ : Area-under-the-concentration-time-curve extrapolated to infinity; $A \cup C_{\text {Overttmax: }}$ Area-under-the-concentration-time-curve to time of maximum concentration $\left(T_{\max }\right)$ of drug given overtly; $A \cup C_{\text {Reftmax }}$ : Area-under-theconcentration-time-curve to time of maximum concentration $\left(T_{\max }\right)$ of reference product; BMI: Body mass index; Cl: Confidence interval; $C_{\text {max }}$ : Maximum concentration; CV: Coefficient of variation; FDA: Food and Drug Administration; HC: Health Canada; HPLC: High performance liquid chromatography; IBE: Individual bioequivalence; KFSH\&RC: King Faisal Specialist Hospital and Research Center; MSR: Mean square residual error from ANOVA; SD: Standard deviation; $T_{\text {max: }}$ Time of maximum concentration;
\end{abstract} $\wedge$ : Terminal elimination rate constant

\section{Acknowledgments}

Special thanks to participants of the study for their dedication and contribution to research. The superb assistance by the research staff at the Clinical Studies and Empirical Ethics Department is gratefully acknowledged.

\section{Funding}

This study was supported by a research grant (RAC 2101105) from the King Faisal Specialist Hospital \& Research Center, Riyadh, Saudi Arabia to MMH. The funder had no role in the design and conduct of the study; in the collection, management, analysis, and interpretation of the data; or in the preparation, review, or approval of the manuscript.

\section{Availability of data and materials}

The dataset supporting the conclusions of this article is available upon request from $\mathrm{MMH}$.

\section{Authors' contributions}

MMH had full access to all of the data in the study and takes responsibility for the integrity of the data and the accuracy of the data analysis. Study concept and design: MMH. Acquisition, analysis, or interpretation of data: All authors. Drafting of the manuscript: MMH. Critical revision of the manuscript for important intellectual content: All authors. Statistical analysis: MMH. Administrative, technical, or material support: AY, FSS, RH, RAS. All authors read and approved the final manuscript.

\section{Competing interests}

The authors declare that they have no competing interests.

\section{Consent for publication}

Not applicable.

\section{Ethics approval and consent to participate}

A written "consent" (specific to the drug to be administered) was obtained from each volunteer; being told that the study compares the effects of tablets/capsules containing placebo to those containing the particular drug on a new serum marker, that it aims to determine how much of the observed changes in the serum marker is not related to the particular drug, and that they will each receive both the drug and placebo in a random sequence. At the completion of the three studies and after obtaining their monetary compensation, the volunteers were contacted for debriefing on the actual study aim and design and for delayed full consenting.

\section{Publisher's Note}

Springer Nature remains neutral with regard to jurisdictional claims in published maps and institutional affiliations.

Received: 1 March 2017 Accepted: 10 May 2017

Published online: 23 May 2017

\section{References}

1. Tilburt JC, Emanuel EJ, Kaptchuk TJ, Curlin FA, Miller FG. Prescribing "placebo treatments": Results of national survey of US internists and rheumatologists. BMJ. 2008:337:a1983

2. Moerman $D E$, Jonas WB. Deconstructing the placebo effect and finding the meaning response. Ann Intern Med. 2002:136(6):471-6.

3. Kienle GS, Kiene $\mathrm{H}$. The powerful placebo effect: fact or fiction? I Clin Epidemiol. 1997:50(12):1311-8.

4. Hróbjartsson A, Gøtzsche PC. Is the placebo powerless? An analysis of clinical trials comparing placebo with no treatment. N Engl J Med. 2001: 344(21):1594-602.

5. McRae C, Cherin E, Yamazaki TG, Diem G, Vo AH, Russell D, et al. Effects of perceived treatment on quality of life and medical outcomes in a doubleblind placebo surgery trial. Arch Gen Psychiatry. 2004;61(4):412-20.

6. Preference Collaborative Review Group. Patients' preferences within randomised trials: systematic review and patient level meta-analysis. BMJ. 2008;337:a1864.

7. Hammami MM, Al Gaai E, Alvi S, Hammami MB. Interaction between drug and placebo effect: across over balanced placebo design. Trials. 2010:11:110.

8. Hammami MM, Hammami S, Al-Swayeh R, Al-Gaai E, Farah FA, De Padua SJ. Drug* placebo interaction effect may bias clinical trials interpretation: hybrid balanced placebo and randomized placebo-controlled design. BMC Med Res Methodol. 2016;16:166.

9. Krogsbøll LT, Hróbjartsson A, Gøtzsche PC. Spontaneous improvement in randomised clinical trials: meta-analysis of three-armed trials comparing no treatment, placebo and active intervention. BMC Med Res Methodol. 2009:9:1.

10. Sayette MA, Breslin FC, Wilson GT, Rosenblum GD. An evaluation of the balanced placebo design in alcohol administration research. Addict behave. 1994;19(3):333-42.

11. Schneider R, Grüner M, Heiland A, Keller M, Kujanora Z, Peper M, et al. Effects of expectation and caffeine on arousal, well-being, and reaction time. Int J Behav Med. 2006;13(4):330-9.

12. Dunne SS, Dunne CP. What do people really think of generic medicines? A systematic review and critical appraisal of literature on stakeholder perceptions of generic drugs. BMC Med. 2015;13:173.

13. Piguet V, D'Incau S, Besson M, Desmeules J, Cedraschi C. Prescribing generic medication in chronic musculoskeletal pain patients: an issue of representations, trust, and experience in a Swiss cohort. PLoS One. 2015 10(8):e0134661.

14. Lund $K$, Vase L, Petersen GL, Jensen TS, Finnerup NB. Randomised controlled trials may underestimate drug effects: balanced placebo trial design. PLoS One. 2014;9(1):e84104.

15. Kirsch I, Moore TJ, Scoboria A, Nicholls SS. The emperor's new drugs: An analysis of antidepressant medication data submitted to the U.S. Food and Drug Administration. Prevention \& Treatment. 2002; 5(1): ArtID 23. http:// psycnet.apa.org/journals/pre/5/1/23a/. Accessed 1 May 2017.

16. Wolf S. The pharmacology of placebos. Pharmacol Rev. 1959;11:689-704.

17. Gundersen H, Specht K, Grüner R, Ersland L, Hugdahl K. Separating the effects of alcohol and expectancy on brain activation: an fMRI working memory study. Neurolmage. 2008:42(4):1587-96.

18. Davit B, Braddy AC, Conner DP, Yu LX. International guidelines for bioequivalence of systemically available orally administered generic drug products: a survey of similarities and differences. APPS J. 2013;15(4):974-90.

19. Chen M-L, Shah VP, Crommelin DJ, Shargel L, Bashaw D, Bhatti M, et al. Harmonization of regulatory approaches for evaluating therapeutic equivalence and interchangeability of multisource drug products: workshop summary report. AAPS J. 2011;13(4):556-64.

20. Kaushal N, Singh SK, Gulati M, Vaidya Y, Kaushik M. Study of regulatory requirements for the conduct of bioequivalence studies in US, Europe, Canada, India, ASEAN and SADC countries: Impact on generic drug substitution. J App Pharm Sci. 2016;6(4):206-22.

21. Guidance for industry. Statistical approaches to establishing bioequivalence. US DHHS, FDA, CDER; 2001. https://www.fda.gov/downloads/Drugs/ Guidances/ucm070244.pdf. Accessed 1 May 2017. 
22. Hsuan FC. Some statistical considerations on the FDA draft guidance for individual bioequivalence. Stat Med. 2000;19(20):2879-84.

23. Buehler G. History of bioequivalence for critical dose drugs. http://www.fda. gov/downloads/\%20AdvisoryCommittees/\%E2\%80\%A6/UCM209319.pdf. Accessed 1 May 2017.

24. Lecaillon J, Hirtz J, Schoeller J, Humbert G, Vischer W. Pharmacokinetic comparison of cefroxadin (CGP 9000) and cephalexin by simultaneous administration to humans. Antimicrob Agents Chemother. 1980;18(4):65660 .

25. Deppermann K, Lode H, Hoffken G, Tschink G, Kalz C, Koeppe P. Influence of ranitidine, piprenzepine, and aluminum magnesium hydroxide on the bioavailability of various antibiotics, including amoxicillin, cephalexin, doxycycline, and amoxicillin-clavulanic avid. Antimicrob Agents Chemother. 1989;33(11):1901-7.

26. Schettler T, Paris S, Pellett M, Kidner S, Wilkinson D. Comparative pharmacokinetics of two fast-dissolving oral ibuprofen formulations and a regular-release ibuprofen tablet in healthy volunteers. Clin Drug Investig. 2001;21(1):73-8.

27. Portoles A, Puerro M, Terleira A, Rodriguez A, Caturla MC, Fernandez N, et al. A new high-absorption-rate paracetamol 500-mg formulation: a comparative bioavailability study in healthy volunteers. Curr Ther Res Clin Exp. 2003;64(7):401-11

28. Dickert N, Grady C. What's the price for a research subject? Approaches to payment for research participation. N Engl J Med. 1999;341(3):198-203.

29. Miller FG, Wendler D, Swartzman LC. Deception in research on the placebo effect. PLoS Med. 2005;2(9):e262. doi:10.1371/journal.pmed.0020262.

30. United States Department of Health and Human Services. Institutional Review Board Guidebook (Chapter V): Biomedical and Behavioral Research An Overview. https://archive.hhs.gov/ohrp/irb/irb_chapter5.htm. Accessed 1 May 2017.

31. American Psychological Association. Ethical Principles of Psychologists and Code of Conduct (including 2010 and 2016 Amendments). http://www.apa. org/ethics/code/. Accessed 1 May 2017.

32. Council for International Organizations of Medical Sciences, International Ethical Guidelines for Health-related Research Involving Humans. Council for International Organizations of Medical Sciences (CIOMS). http://cioms.ch/ ethical-guidelines-2016/WEB-CIOMS-EthicalGuidelines.pdf. Accessed 1 May 2017.

33. Hussein RF, Hammami MM. Determination of cephalexin level and stability in human plasma by fully validated rapid HPLC analysis. WJPPS. 2014;3(12): 20-31.

34. Alvi SN, Yusuf A, Al Gaai E, Hammami MM. Rapid determination of ibuprofen concentration in human plasma by high performance liquid chromatography. WJPPS. 2014;3(8):1767-77.

35. Alswayeh R, Alvi SN, Hammami MM. Rapid determination of acetaminophen levels in human plasma by high performance liquid chromatography. Am J PharmTech Res. 2016;6(3):710-9.

36. Randomization.com. Dallal GE. http://www.randomization.com/. Accessed 1 May 2017.

37. Nuzzo R. Scientific method: statistical errors. Nature. 2014;506(7487):150-2. doi:10.1038/506150a.

38. Sterne JAC, Smith GD. Sifting the evidence-what's wrong with significance tests? BMJ. 2001;322(7280):226-31.

39. Lilford RJ, Braunholtz DA. Is the placebo powerless: Correspondence to the Editor. N Engl J Med. 2001;345(17):1277-8.

40. Siegel S. Explanatory mechanisms for placebo effects: Pavlovian conditioning. In: Guess H, Kleinman A, Kusek J, Engel L, editors. The Science of the Placebo: Toward an interdisciplinary agenda. London, England: BMJ Publishing Group; 2002. p. 133-57.

41. Jiang W, Makhlouf F, Schuirmann DJ, Zhang X, Zheng N, Conner D, et al. A bioequivalence approach for generic narrow therapeutic index drugs: evaluation of the reference-scaled approach and variability comparison criterion. AAPS J. 2015;17(4):891-901.

42. Henney JE. Review of generic bioequivalence studies. JAMA. 1999;282(21):1995

43. Davit BM, Nwakama PE, Buehler GJ, Conner DP, Haider SH, Patel DT, et al. Comparing generic and innovator drugs: a review of 12 years of bioequivalence data from the United States Food and Drug Administration. Ann Pharmacother. 2009;43(10):1583-97.

44. Birkett DJ. Generics-equal or not? Aust Prescr. 2003;26(4):85-7.
45. Yu Y, Teerenstra S, Neef C, Burger D, Maliepaard M. Investigation into the interchangeability of generic formulations using immunosuppressants and a broad selection of medicines. Eur J Clin Pharmacol. 2015;71(8):979-90.

46. Tóthfalusi L, Endrényi L, Chow SC. Statistical and regulatory considerations in assessments of interchangeability of biological drug products. Eur $\mathrm{J}$ Health Econ. 2014;15(Suppl 1):S5-11.

47. Johnston A. Equivalence and interchangeability of narrow therapeutic index drugs in organ transplantation. Eur J Hosp Pharm Sci Pract. 2013; 20(5):302-7.

48. Yim DS. Simulation of the AUC changes after generic substitution in patients. J Korean Med Sci. 2009;24(1):7-12.

49. Ting TY, Jiang W, Lionberger R, Wong J, Jones JW, Kane MA, et al. Generic lamotrigine versus brand-name Lamictal bioequivalence in patients with epilepsy: A field test of the FDA bioequivalence standard. Epilepsia. 2015; 56(9):1415-24

\section{Submit your next manuscript to BioMed Central and we will help you at every step:}

- We accept pre-submission inquiries

- Our selector tool helps you to find the most relevant journal

- We provide round the clock customer support

- Convenient online submission

- Thorough peer review

- Inclusion in PubMed and all major indexing services

- Maximum visibility for your research

Submit your manuscript at www.biomedcentral.com/submit 